\title{
The role of central and peripheral vision in postural control during walking
}

\author{
BENOÎT G. BARDY \\ University of the Mediterranean, Marseille, France \\ and \\ WILLIAM H. WARREN, JR., and BRUCE A. KAY \\ Brown University, Providence, Rhode Island
}

\begin{abstract}
Three hypotheses have been proposed for the roles of central and peripheral vision in the perception and control of self-motion: (1) peripheral dominance, (2) retinal invariance, and (3) differential sensitivity to radial flow. We investigated postural responses to optic flow patterns presented at different retinal eccentricities during walking in two experiments. Oscillating displays of radial flow $\left(0^{\circ}\right.$ driver direction), lamellar flow $\left(90^{\circ}\right)$, and intermediate flow $\left(30^{\circ}, 45^{\circ}\right)$ patterns were presented at retinal eccentricities of $0^{\circ}, 30^{\circ}, 45^{\circ}, 60^{\circ}$, or $90^{\circ}$ to participants walking on a treadmill, while compensatory body sway was measured. In general, postural responses were directionally specific, of comparable amplitude, and strongly coupled to the display for all flow patterns at all retinal eccentricities. One intermediate flow pattern $\left(45^{\circ}\right)$ yielded a bias in sway direction that was consistent with triangulation errors in locating the focus of expansion from visible flow vectors. The results demoristrate functionally specific postural responses in both central and peripheral vision, contrary to the peripheral dominance and differential sensitivity hypotheses, but consistent with retinal invariance. This finding emphasizes the importance of optic flow structure for postural control regardless of the retinal locus of stimulation.
\end{abstract}

In the last 25 years, various roles have been ascribed to central and peripheral vision for the perception and control of self-motion. Three basic positions have emerged. (1) The peripheral dominance hypothesis states that peripheral vision dominates the perception of self-motion, whereas central vision dominates the perception of object motion (Dichgans \& Brandt, 1978, p. 777). On this view, the retinal locus of stimulation plays a causal role in determining self-motion. (2) In contrast, the retinal invariance hypothesis states that self-motion and object motion are perceived on the basis of information in optic flow, independent of the eccentricity of stimulation (Crowell \& Banks, 1993; Gibson, 1968). There is thus no functional specialization for self-motion. (3) The functional sensitivity hypothesis, a combination of the two, proposes that self-motion and object motion are perceived on the basis of optical information but that central and peripheral vision are differentially sensitive to the flow patterns that typically fall on these retinal regions (Andersen \& Braunstein, 1985; Stoffregen, 1985; Warren \& Kurtz, 1992). Specifically, central vision is sensitive

Preparation of this article was supported in part by the French Ministère de l'Education Nationale, de la Recherche et de la Technologie and by U.S. National Institutes of Health Grant EY10923. Correspondence should be addressed to B. G. Bardy, the University of Paris-Sud XI, Department of Sports Sciences (STAPS), Bâtiment 335, 91405 Orsay cedex, France (e-mail: benoit.bardy@staps.u-psud.fr)

Accepted by previous editor, Myron L. Braunstein to a variety of radial, rotary, and lamellar flow patterns, whereas peripheral vision is sensitive to lamellar flow but insensitive to radial and rotary flow. In the literature, central vision is typically considered to be the central $30^{\circ}$ of the visual field.

\section{Retinal Sensitivity to Optic Flow}

As described by Gibson (1950), movement of the observer through a rigid environment generates optic flow patterns that are specific to self-motion. Considered on a spherical projection surface surrounding the observer, translation on a straight path produces a radial flow pattern with a focus of expansion in the direction of selfmotion, grading into lamellar (parallel) flow in the perpendicular direction, and finally a focus of contraction in the opposite direction. In contrast, observer rotation produces global rotary flow about the axis of rotation, grading into lamellar flow in the perpendicular direction. The flow pattern on the retina, however, depends not only on the type of self-motion but also on the direction of gaze and eye movements. For example, if the observer looks in the direction of translation (point A in Figure 1a), the retinal flow pattern will be radial in central vision and increasingly lamellar in peripheral vision. Looking off to one side near the horizon (point B) will yield a similar radial flow pattern in the periphery. Thus, given that radial or lamellar flow can appear at any eccentricity, retinal invariance would be advantageous. On the other hand, if a point nearer to the observer is tracked with a pursuit eye movement (point $\mathrm{C}$ in Figure $1 \mathrm{~b}$ ), the retinal flow pattern 
(a)

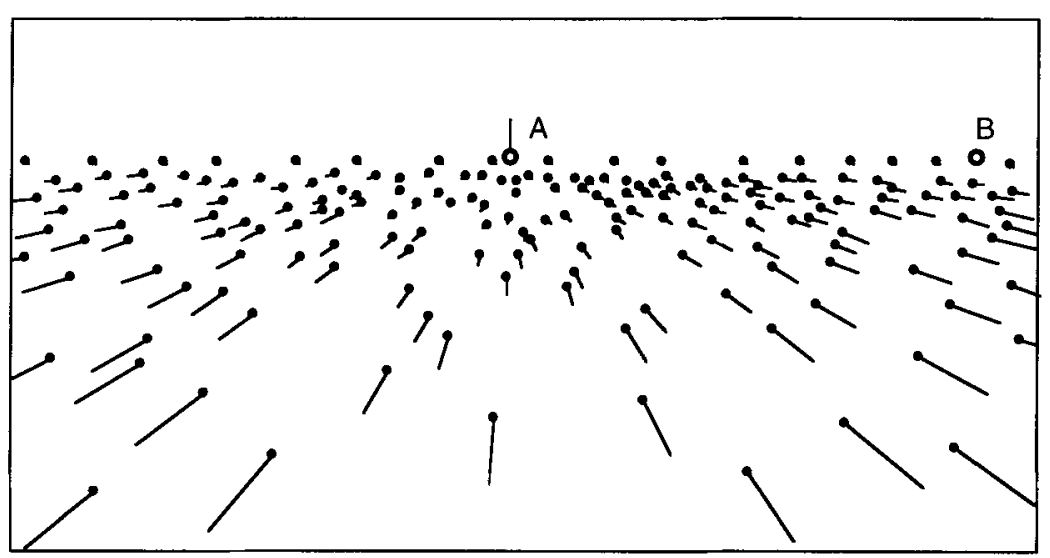

(b)

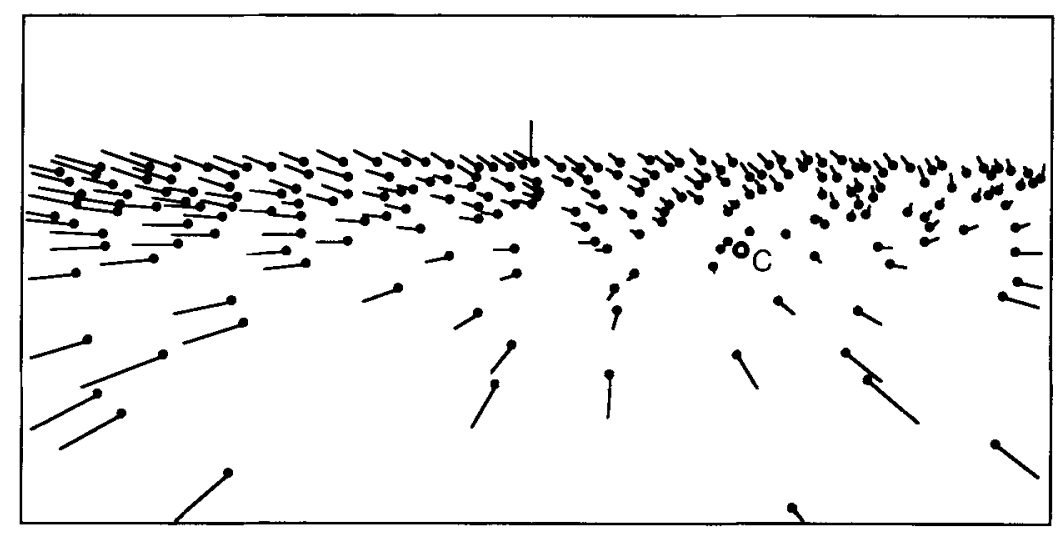

Figure 1. Retinal velocity field produced by translation parallel to a ground plane while (a) fixating in the direction of self-motion at point $A$ or near the horizon at point $B$, and (b) fixating a spot on the ground plane at point $C$, which induces a pursuit eye rotation. Vertical line indicates heading direction.

in the periphery tends to be locally lamellar, while in central vision it is more complex and depends on the 3D structure of the scene. In general, radial and rotary flow patterns are thus less common in the periphery.

The data on retinal sensitivity are mixed (see Warren \& Kurtz, 1992, for a critical review). Initial results favoring the peripheral dominance hypothesis came from the study of vection, or the subjective sensation of selfmotion. Brandt, Dichgans, and Koenig (1973) first reported that circular (yaw) vection was easily obtained with $30^{\circ}$-diameter displays when they were presented $45^{\circ}$ to $75^{\circ}$ in the periphery, but not when they were presented centrally. Similar results were subsequently reported for roll vection (Held, Dichgans, \& Bauer, 1975) and linear vection (Berthoz, Pavard, \& Young, 1975). However, more recent research has shown that vection can also be obtained in central vision. Using radial flow patterns simulating motion through a 3-D cloud of dots, Andersen and Braunstein (1985) reported linear vection with only $7.5^{\circ}$ central stimulation, and Delorme and Martin (1986) elicited linear vection in an oscillating room with $40^{\circ}$ central stimulation. Most convincingly, Post
(1988) found equal circular vection in central and peripheral vision with a $30^{\circ}$ display, contrary to the peripheral dominance hypothesis.

The peripheral dominance hypothesis initially appeared to generalize to postural responses during standing (Amblard \& Carblanc, 1980; Lestienne, Soechting, \& Berthoz, 1977). But subsequent experiments found that postural sway could be elicited by central stimulation as well, using both radial and lamellar flow as small as $15^{\circ}$ in diameter (Andersen \& Dyre, 1989; Delorme \& Martin, 1986; Nougier, Bard, Fleury, \& Teasdale, 1997). Paulus, Straube, and Brandt (1984) even found that spontaneous postural sway when one is viewing a textured wall was substantially lower with $30^{\circ}$ of central vision than with $30^{\circ}$ of peripheral vision, owing to information from binocular convergence. In a suggestive set of experiments with a moving room, Stoffregen $(1985,1986)$ reported that both radial and lamellar flow patterns $\left(60^{\circ}\right.$ diameter $)$ induced significant postural sway when presented centrally, whereas only lamellar flow was effective $90^{\circ}$ in the periphery. This interaction led Stoffregen to propose that the central retina is sensitive to both types of flow but 
that the peripheral retina is insensitive to radial flow for postural control - the functional sensitivity hypothesis.

In the context of judging the direction of self-motion, Warren and Kurtz (1992) also found that heading accuracy was higher when the focus of expansion (FOE) appeared at the fovea as opposed to the periphery, and they interpreted this result in line with the functional sensitivity hypothesis. However, their displays confounded the retinal eccentricity of the FOE with its position in the display. Crowell and Banks (1993) studied the discrimination of successive flow fields and found a similar four-fold advantage when the FOE was at the fovea, but otherwise constant performance as a function of retinal eccentricity, with a range of radial to lamellar flow patterns. In an ideal observer analysis (Crowell \& Banks, 1996), they concluded that radial flow has a small foveal advantage, and that radial flow is extracted an order of magnitude less efficiently than lamellar flow. Otherwise, the efficiency for both radial and lamellar flow is constant across eccentricity, contrary to any cortical magnification factor but consistent with retinal invariance. In addition, Stoffregen and Riccio (1990) reported that head-dodging responses to radial flow patterns specifying a looming object were equally accurate in central and peripheral $\left(90^{\circ}\right)$ vision.

Even though vection, postural control, and heading perception are to some extent dissociable (Warren \& Kurtz, 1992), it is likely that they share some visual processes in common. With regard to central vision, the literature discussed above offers converging evidence from all three phenomena for central sensitivity to both radial and lamellar flow, leading to the rejection of simple peripheral dominance. With regard to peripheral vision, sensitivity to radial flow has been demonstrated for the cases of heading and looming, but not for vection or posture. Thus, the question of retinal invariance or differential sensitivity remains unresolved. In the present study, we examine it for the case of postural control during locomotion.

\section{Visual Control of Posture During Locomotion}

To keep balance during locomotion, observers must make adaptive postural adjustments while maintaining forward progression. As in the case of standing posture, optic flow at the eye of a walking observer contains information that specifies the appropriate compensatory responses. By manipulating a visual "driver" display for an observer on a treadmill, we have recently shown that both radial flow and motion parallax information are used to control postural sway during walking (Bardy, Warren, \& Kay, 1996; Warren, Kay, \& Yilmaz, 1996). In general, compensatory sway is functionally specific to the specified disturbance; it is (1) directionally specific or in the direction specified by the flow pattern, (2) isotropic or of comparable amplitude in all driver directions, and (3) strongly coupled to the visual driver with a high cross-correlation in all directions.

The present experiments were designed to test whether these postural responses depend on the retinal eccentricity of the display. We presented radial and lamellar flow patterns in central and peripheral vision to subjects walking on a treadmill, and we measured their postural sway. The retinal invariance hypothesis predicts that postural responses will be functionally specific at all eccentricities. In contrast, the differential sensitivity hypothesis predicts functionally specific responses to both radial and lamellar flow in central vision, but only to lamellar flow in peripheral vision. Finally, the peripheral dominance hypothesis predicts functionally specific responses with peripheral but not central stimulation. The results are consistent with retinal invariance.

\section{EXPERIMENT 1}

Displays $\left(22^{\circ}\right.$ in diameter) of a frontal surface oscillating sinusoidally in depth were presented to observers walking on a treadmill, while postural sway was estimated from a marker on the neck. In Experiment 1, we tested two flow patterns corresponding to postural sway in two directions (see Figure 2): a radial flow pattern with the FOE at the center of the display $\left(0^{\circ}\right.$ flow), and an intermediate flow pattern with an implicit FOE $30^{\circ}$ from the center of the display $\left(30^{\circ}\right.$ flow $)$. These were presented in both central vision $\left(0^{\circ}\right.$ eccentricity) and peripheral vi$\operatorname{sion}\left(30^{\circ}\right.$ eccentricity). The displays included an oscillatory component corresponding to postural sway but not a constant-velocity component corresponding to forward progression, for previous results showed that postural responses are similar with or without the latter (Warren et al., 1996). The range of flow patterns and retinal eccentricities was extended in Experiment 2.

\section{Method}

Subjects. Eight subjects, 20 to 30 years of age, were paid to participate in the experiment. They included 7 graduate students and the first author, who was the only one informed about the aim of the study.

Apparatus. Displays depicted a random-patch frontal wall that oscillated in depth (the visual driver), simulatıng a sinusoidal os-

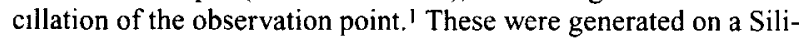
con Graphics Iris 4D/310 VGX workstation at a frame rate of $60 \mathrm{~Hz}$ and presented on a rear-projection screen $(2.94 \mathrm{~m}$ horizontal $[\mathrm{H}] \times$ $2.17 \mathrm{~m}$ vertical $[\mathrm{V}]$ ) using a BARCO Graphics 800 video projector with a $60-\mathrm{Hz}$ refresh rate. Each subject viewed the screen from a distance of approximately $1.2 \mathrm{~m}$ while walking on a motor-driven treadmill (Quinton Q-55, $0.5 \times 1.3 \mathrm{~m}$ belt) at a constant speed of $1 \mathrm{~m} / \mathrm{sec}$. Image resolution was $1,280 \mathrm{H} \times 1,024 \mathrm{~V}$ pixels. The square wall consisted of 256 white squares (each $1.5 \mathrm{~cm}^{2}$ ) in random positions and orientations on a blue background and subtended a mean visual angle of $\theta=22^{\circ}$ over one cycle of oscillation, equivalent to a 0.5 -m-diameter surface at the screen distance of $1.2 \mathrm{~m}$. It oscillated in depth at a frequency of $f=0.25 \mathrm{~Hz}$, with a peakto-peak amplitude of either $A=6.4 \mathrm{~cm}$ (total change in visual angle of $1.2^{\circ}$, mean expansion rate over one half-cycle of $\dot{\theta}, \theta=$ $0.026 / \mathrm{sec}$ ) or $A=10.7 \mathrm{~cm}$ (change of $2.0^{\circ}$, mean expansion rate of $0.044 / \mathrm{sec}$ ). The subject's field of view was restricted to the screen by a head-mounted sport mask.

Design and Procedure. Two eccentricity conditions were crossed with two flow conditions (Figure 2$)$. In the central condition $\left(0^{\circ}\right.$ eccentricity), the treadmill was oriented perpendicular to the screen and subjects were instructed to look straight ahead, ${ }^{2}$ so the center of the display was presented in central vision. In the peripheral condition $\left(30^{\circ}\right.$ eccentricity), the treadmill was rotated $30^{\circ}$ to the left, so 
(a)

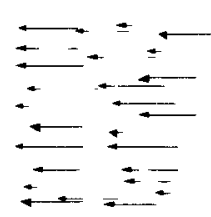

$$
\begin{aligned}
& +2+= \\
& 5+5 \\
& =-5 \\
& =5
\end{aligned}
$$

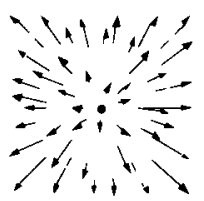

(b)

Lamellar

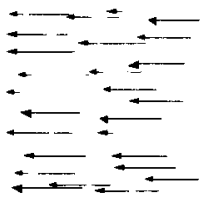

Intermediate

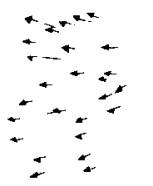

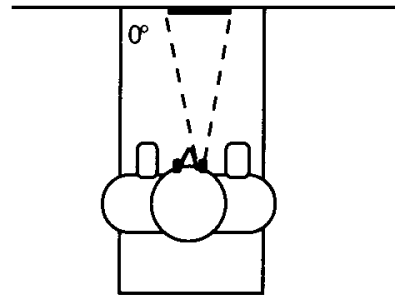

Central
Radial
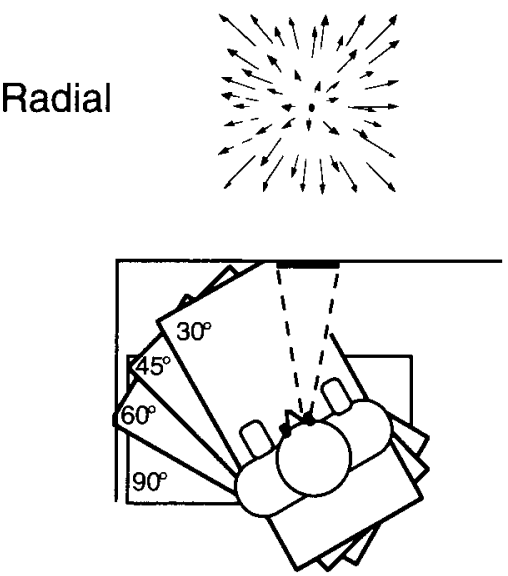

Peripheral

Figure 2. Schematic of experimental conditions: (a) central condition $\left(0^{\circ}\right.$ eccentricity), and (b) peripheral conditions ( $30^{\circ}$ eccentricity in Experiment 1; $45^{\circ}, 60^{\circ}$, and $90^{\circ}$ eccentricity in Experiment 2). Flow conditions included radial $\left(0^{\circ}\right.$ flow), intermediate $\left(30^{\circ}\right.$ or $45^{\circ}$ flow), and lamellar $\left(90^{\circ}\right.$ flow).

that the same display was presented in the right hemifield. In this case subjects looked at a square fixation marker $\left(0.1^{\circ}\right.$ diameter $)$ straight ahead on the screen at a distance of $1.4 \mathrm{~m}$. The $0^{\circ}$ flow condition presented a radial flow pattern produced by oscillation of the eyepoint along an axis perpendicular to the screen (a driver direction of $\alpha=0^{\circ}$ ), so that the FOE was at the center of the display. The $30^{\circ}$ flow condition presented an intermediate flow pattern that included both radial and lamellar components, produced by oscillation of the eyepoint along a diagonal axis (a driver direction of $\alpha=$ $30^{\circ}$ ), so the implicit FOE was $30^{\circ}$ to the right of display's center and, on the average, $19^{\circ}$ to the right of the display's edge. A third variable of driver amplitude was also manipulated $(A=6.4 \mathrm{~cm}$ and $10.7 \mathrm{~cm}$ peak to peak), for a total of eight conditions.

The subjects walked on the treadmill while viewing the screen monocularly with the right eye. They were instructed to "go with the flow" and not consciously antıcipate or resist the display motion. A familiarization period included 3 min of practice walkıng on the treadmill with the room lights on and no display, followed by $3 \mathrm{~min}$ of practice walking with a display of a static wall, and 3 min of practice with an oscillating wall. There were 6 trials in each of the eight test conditions, plus 6 control trials (three in each eccentricity con- dition) usıng a display of the same random-patch wall with no oscllation, for a total of 54 trials in a $1-\mathrm{h}$ session. On each trial, the display was presented for $20 \mathrm{sec}$ to allow the subject to achieve a steady state, followed by a $30-\mathrm{sec}$ data sample. Trials were blocked by eccentricity condition; experimental trials in each of the flow conditions and control trials were all randomized withın each block. Block presentation was counterbalanced for order between subjects.

Data acquisition and analysis. As a measure of body sway, we recorded the motion of a $1.5-\mathrm{cm}$ reflective marker mounted on the left side of the subject's neck, in a locatıon that minimized the effects of neck flexion and extension. Although this did not allow detalled analysis of trunk movement, a neck marker was sufficient to determine the direction and amplitude of the overall sway response. Marker position in three dimensions was measured with a two-camera ELITE infrared motion analysıs system (Ferrigno \& Pedott1, 1985) at a sampling rate of $100 \mathrm{~Hz}$. The two cameras were placed on the subject's left side, and measurements were accurate to $1 \mathrm{~mm}$ in the saggital plane and $2 \mathrm{~mm}$ along the lateral body axis. A $30-\mathrm{sec}$ data sample was initiated by a signal from the graphics workstation, so that the relative phase between the display and postural sway could be determined. 
For each trial, the time series of body sway was fit with a cosine at the driver frequency, leaving amplitude and phase as free parameters; the same cosine was fit to the control trials as a baseline measure. As is noted in Warren et al. (1996), this method gives results identical to performing a discrete Fourier transform at the driver frequency alone. Because sway could occur in any direction, this cross-correlation was computed at $2^{\circ}$ intervals in the horizontal plane and the direction with the maximal sway amplitude was determined. We used this iterative method because the function relating sway amplitude to direction is complicated to infer from responses in two cardinal directions (see Granat, Barnett, Kirkwood, \& Andrews, 1991). This method was successully applied previously (Bardy et al., 1996; Warren et al., 1996). The resulting multiple $R$ value provided an estimate of the overall strength of the coupling between the driver and sway. ${ }^{3}$ Thus, the dependent variables were (1) the direction $\alpha^{\prime}$ having the maximal amplitude of sway, (2) the peak-to-peak amplitude $A_{\text {max }}^{\prime}$ of sway in that direction, (3) the cross-correlation $R$ between the driver and sway in that direction, and (4) the phase angle $\phi$ between driver and sway. Standard circular statistics (Batschelet, 1981) were used for computing means of direction and phase as well as relevant inferential statistics. Further methodological details are described in Bardy et al. (1996) and Warren et al. (1996).

\section{Results and Discussion}

The mean direction and amplitude of sway in each eccentricity and flow condition are plotted in Figure 3, and detailed results appear in Table 1 . The circumference in Figure 3 represents the direction of sway with respect to the display (e.g., $0^{\circ}$ is the center of the display, the predicted sway direction with $0^{\circ}$ radial flow); the direction of sway with respect to the body (and hence the retinal eccentricity) is indicated by the labels $\mathrm{A} / \mathrm{P}$ for anterior/ posterior and $\mathrm{L} / \mathrm{R}$ for left/right. This way of plotting the data means that, if responses are functionally specific to the flow pattern regardless of eccentricity, central and peripheral plots will look similar.

The displays elicited postural sway in all 8 subjects, as is shown by significantly greater cross-correlations between driver and sway in experimental trials than in control trials ( $t$ tests for each subject, $p<.05$ or better). Overall, the mean cross-correlation was $R=.69(S D=.20$, $N=384)$ on experimental trials and $R=.16(S D=.05$, $N=48$ ) on control trials $[t(430)=9.14, p<.0001]$. In addition, the mean amplitude of sway was significantly greater in experimental trials $(11.65 \mathrm{~cm})$ than in control trials $(1.26 \mathrm{~cm})[t(430)=10.40, p<.0001]$. These differences were statistically reliable in every condition (Table 1 , columns 6 and 8), indicating a significant postural response to both flow patterns at both eccentricities.

Several points should be noted: First, responses to radial and intermediate flow patterns were directionally specific in both central and peripheral vision. Second, the cross-correlations between driver and sway were similar at both eccentricities. Third, responses were isotropic - that is, sway amplitude was similar in both driver directions - at both eccentricities. Fourth, response amplitude was functionally related to driver amplitude at both eccentricities. However, the overall amplitude of sway was lower in the peripheral than in the central condition. Let us discuss these results in detail.
Direction of sway. As expected, the mean direction of sway closely matched the driver direction in each condition (Table 1, column 4). For every subject, there was a significant circular correlation between driver and sway directions, with a mean of $r=.88(p<.0001, N=384)$. Furthermore, responses for all trials in each condition $(N=48)$ significantly clustered around a mean direction of sway, as shown by Raleigh tests that ranged from $r=$ .77 to $r=.97$, all $p s<.05$ or better. The $95 \%$ confidence interval about this mean direction of sway contained the driver direction in every condition, indicating that subjects swayed in the direction specified by the driver, with only one exception (peripheral, $0^{\circ}$ flow, high amplitude). There was no difference in the mean direction of sway between the central and peripheral conditions, as was shown by a Watson-Williams $F$ test for circular variables $[F(1,382)=2.23, p>.05]$. Although the standard deviation of sway direction tended to be higher in the peripheral condition, the difference was not significant [WatsonWilliams $F(1,6)=3.06, p>.05]$. In sum, $0^{\circ}$ and $30^{\circ}$ flow patterns were reliably used by subjects to control the direction of body sway in both the central and the peripheral conditions.

Coupling strength. The cross-correlation between driver and sway for each condition was in the range $R=$ .55 to .75 (Table 1 , column 8 ), indicating a strong visual coupling in all conditions. To analyze this pattern of results, we performed a four-way repeated measures analysis of variance (ANOVA) (eccentricity $\times$ flow $\times$ driver amplitude $\times$ trials) on $z$-transformed $R$ values. The analysis yielded no effect of eccentricity $[F(1,7)=3.89, p>$ $.05]$ or of flow $[F(1,7)=0.09, p>.05]$, nor were there any interactions. This suggests that the strength of coupling between sway and driver was comparable for flow patterns in both central and peripheral vision. To assess these null results, a power analysis was performed (Keppel, 1982), assuming an acceptible level of power $(1-\beta=$ .80 ) and $\alpha=.05$. For the observed eccentricity effect (a difference in $R$ of $.74-.62=.12$ ) to have reached statistical significance, given the observed variance $\left(S^{2}=\right.$ $1.276), 380$ subjects would have been required. This implies that the risk of making a Type II error was very low, supporting the interpretation that coupling strength was similar at both eccentricities. The observed flow effect was nil (a difference in $R$ of $.68-.67=.01$ ). For the eccentricity $\times$ flow interaction, the present experiment had sufficient power to detect a mean cell difference in $R$ of .14 , given the observed variance $\left(S^{2}=0.087\right)$. This implies that the coupling strengths were similar for both flow patterns at both eccentricities. Finally, there was a main effect of driver amplitude $[F(1,7)=15.37, p<.01]$, indicating that coupling strength was significantly weaker with the smaller driver.

Sway amplitude. The mean peak-to-peak amplitude of body sway in each condition (Table 1, column 6) indicates that responses were isotropic - that is, having highly similar amplitudes in both driver directions. A four-way ANOVA of sway amplitude revealed, first, no effect of 

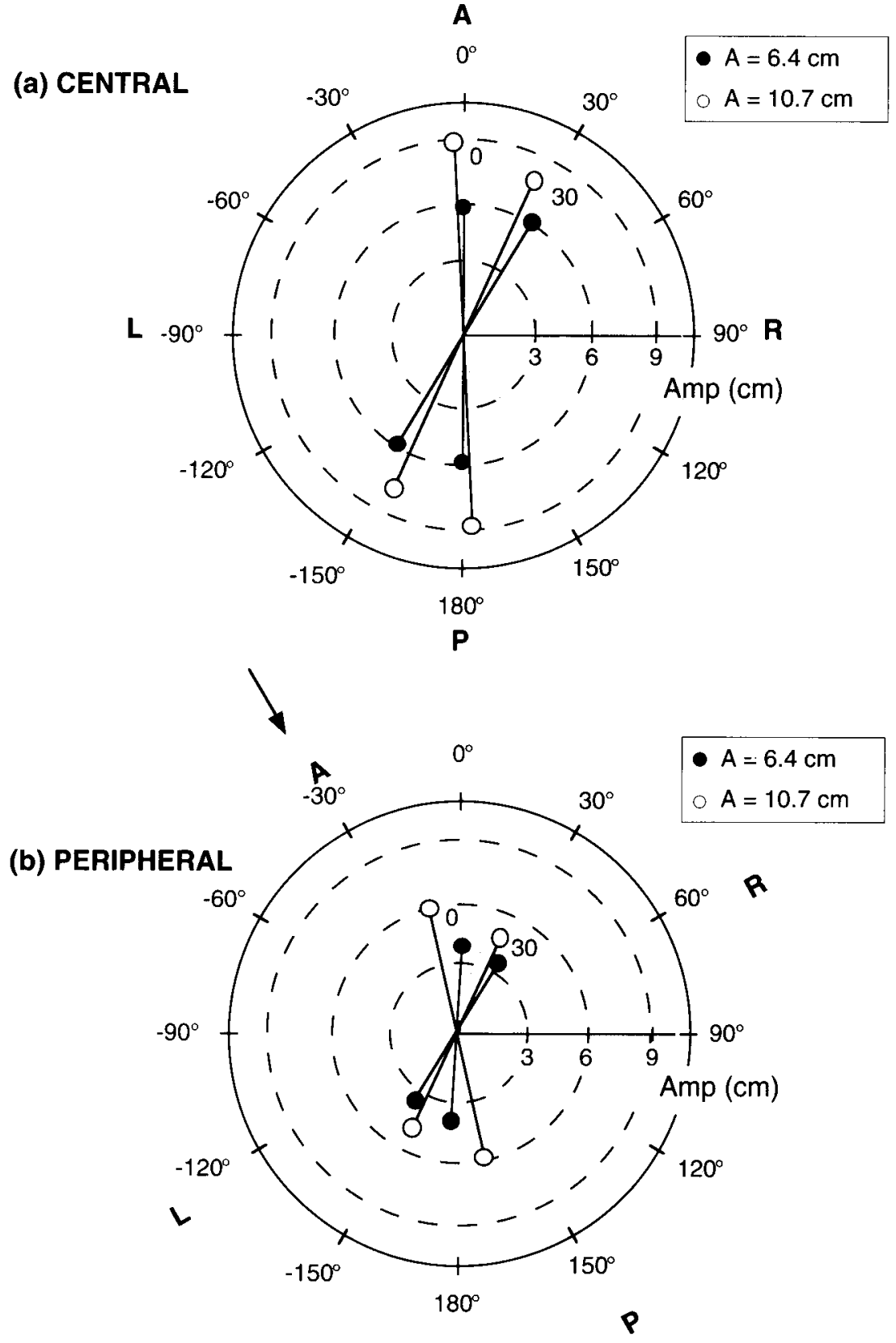

Figure 3. Polar plots representing mean direction and amplitude of body sway in Experiment 1 : (a) central condition $\left(0^{\circ}\right.$ eccentricity), (b) peripheral condition $\left(30^{\circ}\right.$ eccentricity). The radial $\left(0^{\circ}\right.$ flow) and intermediate $\left(30^{\circ}\right.$ flow) conditions are indicated by numbers on the curves. The circumferential coordinates $\left(0^{\circ}\right.$ to $\left.180^{\circ}\right)$ represent sway direction with respect to the center of the display, which appeared at $0^{\circ}$. The participant's body axes are indicated by $A-P$ and $L-R$; in the peripheral condition, the body axes are rotated with respect to the display by $-30^{\circ}$. Arrows indicate the treadmill orientation. Driver frequency $=0.25 \mathrm{~Hz}$, driver amplitudes $=6.4$ and $10.7 \mathrm{~cm}$.

flow condition $[F(1,7)=3.73, p>.05]$, with a mean of $12.23 \mathrm{~cm}(S D=6.92)$ for $0^{\circ}$ flow and $11.06 \mathrm{~cm}(S D=$ 6.43 ) for the $30^{\circ}$ flow. A power analysis showed that for the observed flow effect (a difference of $1.17 \mathrm{~cm}$ ) to have reached significance, 400 subjects would have been re- quired, given the observed variance $\left(S^{2}=34.78\right)$. The risk of a Type II error was thus very low, implying that sway amplitude was similar in both flow conditions. The lack of a flow $\times$ eccentricity interaction $[F(1,7)=.48$, $p>.05]$, is consistent with isotropic responses in both 
Table 1

Mean Postural Sway and Standard Deviation by Condition in Experiment 1

\begin{tabular}{|c|c|c|c|c|c|c|c|c|c|c|c|}
\hline \multirow{2}{*}{$\begin{array}{c}\text { Retinal } \\
\text { Eccentricity }\end{array}$} & \multirow{2}{*}{$\begin{array}{c}\text { Flow } \\
\text { conditions }\end{array}$} & \multirow{2}{*}{$\begin{array}{c}\text { Driver } \\
\text { Amp }(\mathrm{cm})\end{array}$} & \multicolumn{2}{|c|}{$\alpha^{\prime}(\mathrm{deg})$} & \multicolumn{2}{|c|}{$A_{\text {max }}^{\prime}(\mathrm{cm})$} & \multicolumn{2}{|c|}{$R$} & \multicolumn{2}{|c|}{$\phi(\mathrm{deg})$} & \multirow{2}{*}{$\begin{array}{l}\text { Raleigh } \\
\text { Phase } r\end{array}$} \\
\hline & & & $M$ & $S D$ & $M$ & $S D$ & $M$ & $S D$ & $M$ & $S D$ & \\
\hline \multirow[t]{4}{*}{ Central $\left(0^{\circ}\right)$} & $0^{\circ}$ & 6.4 & $-0.25^{*}$ & 31.42 & $11.62^{*}$ & 5.44 & $.70^{*}$ & .22 & $-39.00^{*}$ & 36.50 & $.80^{*}$ \\
\hline & & 10.7 & $2.89 *$ & 21.05 & $17.58^{*}$ & 8.13 & $.77^{*}$ & .30 & $-35.26^{*}$ & 37.81 & $.78^{*}$ \\
\hline & $30^{\circ}$ & 6.4 & $31.97^{*}$ & 29.59 & $12.00^{*}$ & 5.11 & $.72 *$ & .20 & $-46.63^{*}$ & 31.81 & $.85^{*}$ \\
\hline & & 10.7 & $24.35^{*}$ & 33.25 & $15.50^{*}$ & 6.06 & $.77^{*}$ & .21 & $-31.11^{*}$ & 41.44 & $.74^{*}$ \\
\hline \multirow[t]{4}{*}{ Peripheral $\left(30^{\circ}\right)$} & $0^{\circ}$ & 6.4 & $2.96^{*}$ & 48.39 & $7.90^{*}$ & 4.69 & $.57^{*}$ & .15 & $-18.60^{*}$ & 40.73 & $.75^{*}$ \\
\hline & & 10.7 & $-12.22^{*}$ & 44.24 & $11.82^{*}$ & 6.79 & $.69^{*}$ & .18 & $-31.10^{*}$ & 38.25 & $.78^{*}$ \\
\hline & $30^{\circ}$ & 6.4 & $30.46^{*}$ & 56.61 & $7.00^{*}$ & 4.13 & $.55^{*}$ & .17 & -13.84 & 44.31 & $.70^{*}$ \\
\hline & & 10.7 & $25.45^{*}$ & 49.38 & $9.76^{*}$ & 7.32 & $.65^{*}$ & .13 & -6.44 & 47.63 & $.65^{*}$ \\
\hline Grand mean & & & - & - & $11.65^{*}$ & 5.96 & $.69^{*}$ & .20 & $-27.75^{*}$ & 39.81 & $.76^{*}$ \\
\hline Control & & & - & - & 1.26 & 0.59 & .16 & .05 & - & - & - \\
\hline
\end{tabular}

Note-Frequency $(0.25 \mathrm{~Hz})$ is fixed. For $\alpha^{\prime}$, asterisks indicate that the Raleigh test of nonhomogeneity for sway direction was significant. For $A_{\max }^{\prime}$, asterisks indicate that the experimental amplitude was statistically different from the control amplitude. For $R$, asterisks indicate that the driver sway cross-correlation was statistically different between control and experimental trials. For $\phi$, asterisks indicate that the mean phase angle between driver and sway was statistically different from $0^{\circ}$. For Raleigh phase $r$, asterisks indicate that the Raleigh test of nonhomogeneity for phase was statistically significant. ${ }^{*} p<.05$ or better.

central and peripheral vision. Given the observed variance $\left(S^{2}=17.38\right)$, the present experiment had sufficient power to detect a mean cell difference of $3.8 \mathrm{~cm}$. Sway amplitude was $4 \mathrm{~cm}$ greater with the large driver than with the small driver $[F(1,7)=15.38, p<.01]$, with no interactions. This indicates that responses to both flow patterns were functionally linked to the amplitude of the driver at both eccentricities.

The only unexpected finding was that mean sway amplitude was significantly lower in the peripheral condition $(9.12 \mathrm{~cm}, S D=6.00)$ than in the central condition $(14.17 \mathrm{~cm}, S D=6.37)[F(1,7)=14.85, p<.01]$. Note that this effect was due to the retinal eccentricity of the entire display, not the eccentricity of the FOE; when the implicit FOE appeared in the same retinal location $\left(+30^{\circ}\right.$ eccentricity), sway responses were still $4 \mathrm{~cm}$ smaller in the peripheral condition with radial flow $(9.86 \mathrm{~cm})$ than in the central condition with intermediate flow $(13.75 \mathrm{~cm})$ (Newman-Keuls $p<.05$ ). Conversely, however, when the entire display appeared in the same retinal location, there was no effect of the position of the FOE. Thus, the magnitude of sway was independent of the retinal locus of the FOE, but it depended on the retinal locus of the entire display.

Finally, the ANOVAs showed no effect of the trials factor on either sway amplitude $[F(1,7)=.77, p>.05]$ or the cross-correlation $[F(1,7)=.78, p>.05]$, and no sign of adaptation was observed during the experiment. None of the other interactions was significant.

Phase. In each condition, the phase angle between sway and driver was found to be significantly clustered around a mean, with Raleigh tests ranging from $r=.65$ to $r=$ $.85(N=48, p \mathrm{~s}<.05$ or better $)$ indicating a preferred phase angle (Table 1, columns 10 and 12). By convention, a positive value of phase indicates that the head is leading the display, whereas a negative value suggests that the head is following it. Phase angle was similar in the two flow conditions, with means of $-31.18^{\circ}(S D=38.93)$ for $0^{\circ}$ flow and $-25.94^{\circ}(S D=43.84)$ for $30^{\circ}$ flow [WatsonWilliams $F(1,382)=1.30, p>.05]$. This provides converging evidence that sway was driven in a similar manner by different flow patterns. On the other hand, mean phase angle was significantly more negative in central vision $\left(-38.27^{\circ}, S D=37.40\right)$ than in peripheral vision $\left(-18.03^{\circ}, S D=43.55\right)$ [Watson-Williams $F(1,382)=$ $20.48, p<.01]$, indicating that sway lagged farther behind the display oscillation. This was largely due to the two peripheral conditions with intermediate flow, in which mean phase was not significantly different from $0^{\circ}$. In all other conditions, phase was significantly negative (asterisks in Table 1, column 10).

Thus, the results provide evidence that compensatory sway is functionally specific to the structure of optic flow in both central and peripheral vision. In particular, postural sway (1) was directionally specific to the flow pattern, (2) was isotropic in amplitude across driver directions, (3) had similarly strong cross-correlations with the display, and (4) covaried with display amplitude, whether the stimulation appeared centrally or peripherally. This indicates that peripheral vision is sensitive to radial as well as lamellar flow for purposes of postural control, contrary to the functional sensitivity hypothesis. This pattern of results is consistent with retinal invariance, at least out to an eccentricity of $30^{\circ}$.

The only anomalous result was that the amplitude of sway was actually smaller with peripheral than with central stimulation, and that mean phase was closer to zero in the periphery. One possible explanation is that the peripheral eccentricity tested $\left(30^{\circ}\right)$ is adjacent to the "blind spot," located at $15^{\circ}-20^{\circ}$ along the nasal retina (Pirenne, 1967), perhaps making the driver amplitude harder to detect. A more likely possibility is that the lower amplitude is an artifact of using a fixation marker in the peripheral condition only. A stationary fixation point could contribute to the stabilization of posture. In addition, stabilized gaze may allow greater sensitivity to the phase of 
oscillation of the display. Paulus, Straube, Krafczyk, and Brandt (1989, Figure 6) found that a similar monocular fixation spot $\left(0.07^{\circ}\right.$ at $\left.0.4 \mathrm{~m}\right)$ in the dark did not reduce spontaneous standing sway in comparison with an eyes closed condition, but our testing situation induced much greater sway. Both of these confounds were eliminated in the second experiment.

\section{EXPERIMENT 2}

The purpose of Experiment 2 was to extend the range of retinal eccentricities and flow patterns tested. Our initial finding that peripheral vision is sensitive to both radial and intermediate flow patterns appeared to contradict previous reports that radial flow is ineffective in the periphery (Stoffregen, 1985, 1986). However, whereas Stoffregen's peripheral display appeared at a retinal eccentricity of $90^{\circ}$, ours appeared at an eccentricity of only $30^{\circ}$. The area centralis in the human retina is about $6 \mathrm{~mm}$ in diameter and subtends roughly $10^{\circ} 15^{\circ}$ centered on the fovea (Buser \& Imbert, 1987). In the self-motion literature, eccentricities ranging from $20^{\circ}$ to $90^{\circ}$ are often referred to as peripheral. Thus, our display was at the lower end of the peripheral range, and it is possible that responses to radial flow might deteriorate at greater eccentricities. In the present experiment, we tested a larger range of eccentricities $\left(0^{\circ}, 45^{\circ}, 60^{\circ}, 90^{\circ}\right)$.

Second, previous researchers have compared radial flow with pure lamellar flow, whereas we used an intermediate flow pattern that contained both radial and lamellar components. Thus, in Experiment 2 we also tested a wider range of flow structures, including pure radial $\left(0^{\circ}\right.$ flow), intermediate $\left(45^{\circ}\right.$ flow $)$, and pure lamel$\operatorname{lar}\left(90^{\circ}\right.$ flow $)$, crossed with the four eccentricities. Finally, to eliminate the fixation confound, a fixation point was presented in all conditions.

\section{Method}

The displays, procedure, and data analysis were the same as in Experiment 1, with the following three exceptions. First, the flow conditions included a radial pattern, a lamellar pattern, and an intermediate pattern midway between them. Specifically, the $0^{\circ}$ flow condition was similar to that of Experiment 1, with the FOE at the center of the display, correspondıng to oscillation along an axis perpendicular to the screen. The $45^{\circ}$ flow condition had an intermediate pattern corresponding to oscillation along the main diagonal axis, such that the virtual FOE was $45^{\circ}$ to the right of the center of the display. The $90^{\circ}$ flow condition was a pure lamellar pattern corresponding to oscillation along an axis parallel to the screen, such that the virtual FOE was at $90^{\circ}$. Second, retunal eccentricities included the central condition of Experiment $1\left(0^{\circ}\right.$ eccentricity) and three new peripheral conditions $\left(45^{\circ}, 60^{\circ}, 90^{\circ}\right.$ eccentricity). For the latter conditions, the treadmill was turned to the left, so that the same visual displays were presented in the right hemifield (see Figure 2). To occlude the surrounding room, black curtains were hung on either side of the screen, perpendicular to it. Third, a fixation point $\left(0.1^{\circ}\right.$ diameter $)$ appeared directly in front of the treadmill at eye level in all conditions. For eccentricities of $0^{\circ}$ and $45^{\circ}$, the fixation point was a red dot projected on the screen, whereas for ec- centricities of $60^{\circ}$ and $90^{\circ}$ it was a red LED attached to the curtain on the left side of the screen.

We tested the same driver frequency $(0.25 \mathrm{~Hz})$ and one of the two amplitudes $(A=10.7 \mathrm{~cm})$ from Experiment 1 . The three authors and 9 graduate students at Brown University, 21 to 27 years of age, were paid to participate. None of the students had partıcipated in the first experiment, nor were they informed about the aim of the study. Each subject received 5 experimental trials in each of the 12 conditions plus 5 control trials with a static display for a total of 65 trials. Trials were blocked by eccentricity condition, with condition order counterbalanced across subjects.

\section{Results}

The mean sway direction and amplitude in each condition are presented in Figure 4, and details appear in Table 2. Overall, the mean cross-correlation and sway amplitude were greater in experimental trials $(R=.72$, $\left.A_{\text {max }}^{\prime}=11.29 \mathrm{~cm}, N=720\right)$ than in control trials $(R=$ $\left..15, A_{\text {max }}^{\prime}=1.35 \mathrm{~cm}, N=60\right)[t(778)>11.37, p<.001]$. This difference was statistically reliable in every condition (Table 2, columns 5 and 7), indicating a significant postural response to all flow patterns at all eccentricities.

Four points should be noted about the results. First, the difference in amplitude between central and peripheral conditions observed in Experiment 1 disappeared. Second, sway was strongly coupled to the driver and sway amplitudes were isotropic across driver directions, regardless of retinal eccentricity. Third, the direction of sway closely matched the driver direction in both the radial and the lamellar conditions, but in the intermediate condition it was consistently biased toward the lamellar driver direction. Fourth, subjects followed the driver with about a $20^{\circ}$ phase lag in all conditions. We shall discuss these points in detail.

Sway amplitude. Unlike in Experiment 1, the amplitude of sway was comparable at all eccentricities, with means of $12.59 \mathrm{~cm}(S D=6.33)$ in the $0^{\circ}$ central condition, $10.94 \mathrm{~cm}(S D=6.79)$ in the $45^{\circ}$ peripheral condition, $11.47 \mathrm{~cm}(S D=6.07)$ in the $60^{\circ}$ peripheral condition, and $10.17 \mathrm{~cm}(S D=7.28)$ in the $90^{\circ}$ peripheral condition. In addition, sway amplitude was similar in all driver directions, with means of $10.57 \mathrm{~cm}$ for radial flow, $11.09 \mathrm{~cm}$ for intermediate flow, and $12.22 \mathrm{~cm}$ for lamellar flow. A three-way repeated measures ANOVA of sway amplitude (flow $\times$ eccentricity $\times$ trials) yielded no main effects of eccentricity $[F(3,33)=1.24, p>.05]$ or flow pattern $[F(2,22)=3.12, p>.05]$, and no eccentricity $\times$ flow interaction $[F(6,66)=1.471, p>.05]$. A power analysis showed that for the observed eccentricity effect to have reached significance would have required 526 subjects, given the observed variance $\left(S_{\mathrm{A} \times \mathrm{S}}^{2}=149.73\right)$, and that the observed flow effect would have required 374 subjects, given the observed variance $\left(S^{2} \mathrm{~B} \times \mathrm{S}=55.00\right)$, implying that the risk of a Type II error in either case was low. For the eccentricity $\times$ flow interaction, the present experiment had sufficient power to detect a mean cell difference of $2.8 \mathrm{~cm}$, given the observed variance $\left(S_{\mathrm{A} \times \mathrm{B} \times \mathrm{S}}=\right.$ 45.77). This implies that the sway amplitudes were similar 
(a) CENTRAL

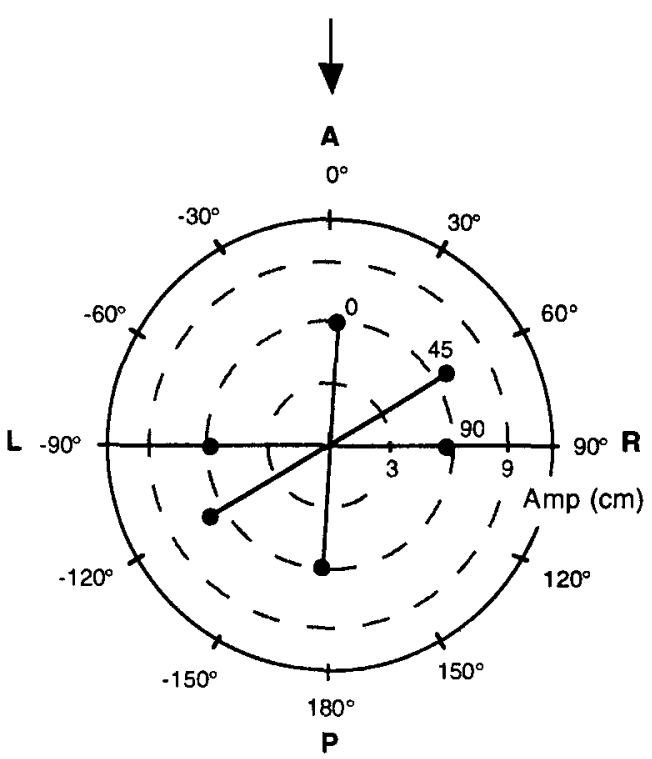

(b) $45^{\circ}$ PERIPHERAL

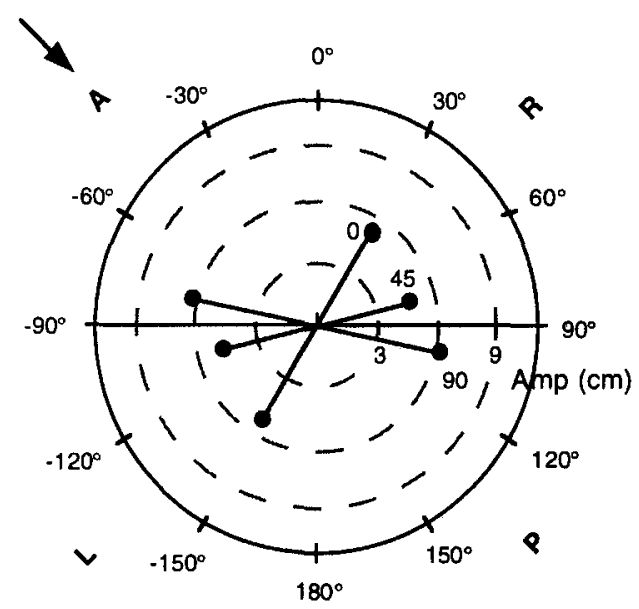

(c) $60^{\circ}$ PERIPHERAL

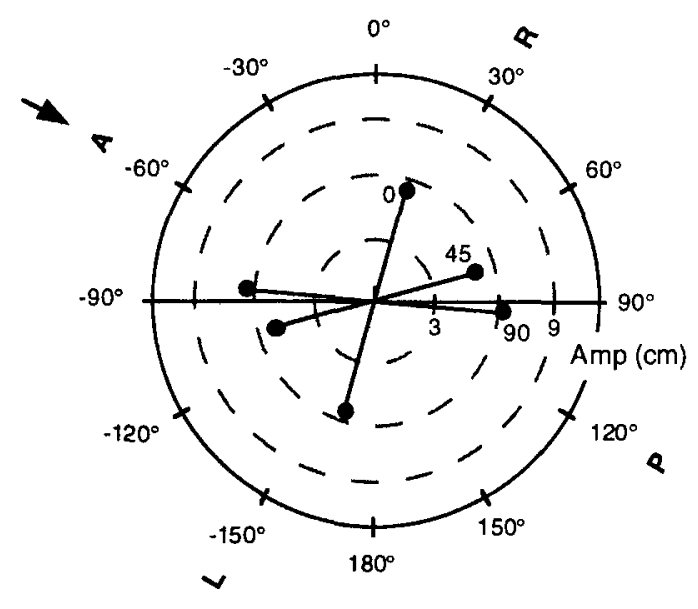

(d) $90^{\circ}$ PERIPHERAL

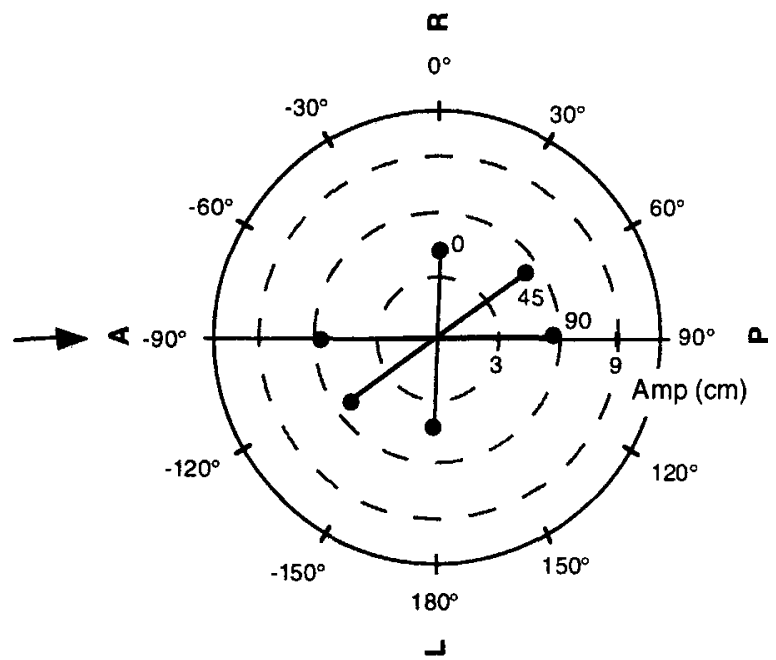

Figure 4. Polar plots representing mean direction and amplitude of body sway in Experiment 2: (a) central condition ( $0^{\circ}$ eccentricity), (b) peripheral $\left(45^{\circ}\right.$ eccentricity), (c) peripheral $\left(60^{\circ}\right.$ eccentricity), and (d) peripheral $\left(90^{\circ}\right.$ eccentricity). The radial $\left(0^{\circ}\right.$ flow), intermediate $\left(45^{\circ}\right.$ flow), and lamellar $\left(90^{\circ}\right.$ flow) conditions are indicated by numbers on the curves. The circumferential coordinates $\left(0^{\circ}\right.$ to $\left.180^{\circ}\right)$ represent sway direction with respect to the center of the display, whereas the subject's body axes are indicated by $A-P$ and $L-R$; in peripheral conditions, the body axes are rotated with respect to the display by $-45^{\circ},-60^{\circ}$, or $-90^{\circ}$. Arrows indicate the orientation of the treadmill. Driver frequency $=0.25 \mathrm{~Hz}$, driver amplitude $=10.7 \mathrm{~cm}$.

in the radial, lamellar, and intermediate flow conditions at all eccentricities. Thus, the lower sway amplitude that was observed in peripheral than in central conditions in Experiment 1 was eliminated in Experiment 2 . It seems likely that this was due to the addition of a fixation point in the central (as well as peripheral) conditions, so that its stabilizing effect was equivalent at all eccentricities.
A general trials effect was observed this time $[F(4,44)=$ $3.49, p<.05]$, accounting for only $1 \%$ of the total variance, indicating a small but significant increase in body sway over the course of a session. Because trials were blocked by eccentricity and counterbalanced for order, this effect appears to have been an adaptation to the displays and testing situation, rather than a specific response 
Table 2

Mean Postural Sway and Standard Deviation by Condition in Experiment 2

\begin{tabular}{|c|c|c|c|c|c|c|c|c|c|c|}
\hline \multirow{2}{*}{$\begin{array}{c}\text { Retinal } \\
\text { Eccentricity }\end{array}$} & \multirow{2}{*}{$\begin{array}{c}\text { Flow } \\
\text { conditions }\end{array}$} & \multicolumn{2}{|c|}{$\alpha^{\prime}(\operatorname{deg})$} & \multicolumn{2}{|c|}{$A_{\text {max }}^{\prime}(\mathrm{cm})$} & \multicolumn{2}{|c|}{$R$} & \multicolumn{2}{|c|}{$\phi(\operatorname{deg})$} & \multirow{2}{*}{$\begin{array}{l}\text { Raleigh } \\
\text { Phase } r\end{array}$} \\
\hline & & $M$ & $S D$ & $M$ & $S D$ & $M$ & $S D$ & $M$ & $S D$ & \\
\hline \multirow[t]{3}{*}{ Central $\left(0^{\circ}\right)$} & $0^{\circ}$ & $3.18^{*}$ & 23.25 & $12.22^{*}$ & 6.74 & $.70^{*}$ & .49 & $-17.77^{*}$ & 40.50 & $.75^{*}$ \\
\hline & $45^{\circ}$ & $59.62 * \dagger$ & 34.78 & $13.38^{*}$ & 6.28 & $8^{*}$ & .43 & $-16.49^{*}$ & 27.02 & $89^{*}$ \\
\hline & $90^{\circ}$ & $89.80^{*}$ & 31.45 & $12.17^{*}$ & 5.98 & $.75^{*}$ & .43 & $-16.71^{*}$ & 36.48 & $.80^{*}$ \\
\hline \multirow[t]{3}{*}{ Peripheral $\left(45^{\circ}\right)$} & $0^{\circ}$ & $29.94 * \dagger$ & 43.55 & $10.53^{*}$ & 5.51 & $.74^{*}$ & .35 & $-18.90^{*}$ & 33.61 & $.83^{*}$ \\
\hline & $45^{\circ}$ & $76.36^{*}+$ & 60.35 & $9.74^{*}$ & 6.48 & $.74 *$ & .36 & $-21.53^{*}$ & 44.10 & $.71^{*}$ \\
\hline & $90^{\circ}$ & $102.37^{*}$ & 52.26 & $12.56^{*}$ & 8.39 & $.71^{*}$ & .32 & $-15.28^{*}$ & 27.02 & $.89^{*}$ \\
\hline \multirow[t]{3}{*}{ Peripheral $\left(60^{\circ}\right)$} & $0^{\circ}$ & $16.08 *$ & 50.11 & $11.07^{*}$ & 5.84 & $.74^{*}$ & .38 & $-21.24^{*}$ & 52.46 & $.58^{*}$ \\
\hline & $45^{\circ}$ & $74.89 * \dagger$ & 48.30 & $10.60^{*}$ & 6.60 & $.75^{*}$ & .39 & $-18.97^{*}$ & 20.80 & $.93^{*}$ \\
\hline & $90^{\circ}$ & $95.24^{*}$ & 48.45 & $12.76^{*}$ & 5.79 & $.74^{*}$ & .39 & $-17.32^{*}$ & 37.40 & $.79^{*}$ \\
\hline \multirow[t]{3}{*}{ Peripheral $\left(90^{\circ}\right)$} & $0^{\circ}$ & $2.40^{*}$ & 48.89 & $8.45^{*}$ & 6.78 & $.59^{*}$ & .43 & $-18.65^{*}$ & 50.30 & $.61^{*}$ \\
\hline & $45^{\circ}$ & $54.19^{*}$ & 57.65 & $10.66^{*}$ & 7.47 & $.69^{*}$ & .45 & $-21.28^{*}$ & 34.86 & $.81^{*}$ \\
\hline & $90^{\circ}$ & $89.33^{*}$ & 42.98 & $11.41^{*}$ & 7.61 & $.67^{*}$ & .48 & $-22.58^{*}$ & 38.71 & $.77^{*}$ \\
\hline Grand mean & & - & - & $11.29^{*}$ & 6.62 & $.72^{*}$ & .41 & $-18.89^{*}$ & 36.93 & $.78^{*}$ \\
\hline Control & & - & & 1.35 & 1.04 & .15 & .07 & - & - & - \\
\hline
\end{tabular}

Note-Frequency $(0.25 \mathrm{~Hz})$ and driver amplitude $(10.7) \mathrm{cm}$ are fixed. For $\alpha^{\prime}$, asterisks indicate that the Raleigh test of nonhomogeneity for sway direction was significant, and daggers indicate that the $95 \%$ confidence interval does not contain the driver direction. For $A_{\max }^{\prime}$, asterisks indicate that the experimental amplitude was statistically different from the control amplitude. For $R$, asterisks indicate that the driver sway cross-correlation was statistically different between control and experimental trials. For $\phi$, asterisks indicate that the mean phase angle between driver and sway was statistically different from zero degrees. For Raleigh Phase $r$, asterisks indicate that the Raleigh test of nonhomogeneity for phase was statistically significant. ${ }^{*} p<.05$ or better.

to a particular flow or eccentricity condition. This was confirmed by the absence of a significant trial $\times$ flow interaction $[F(8,88)=.40, p>.05]$ or a trial $\times$ eccentricity interaction $[F(12,132)=.54, p>.05]$. None of the other interactions were significant.

Coupling strength. The cross-correlations between driver and sway were also high and similar across conditions (Table 2, column 7). A three-way repeated measures ANOVA of $z$-transformed $R$ values (flow $\times$ eccentricity $\times$ trials $)$ indicated no effect of eccentricity $[F(3,33)=$ $2.10, p>.05]$, of flow pattern $[F(2,22)=3.21, p>.05]$, or of any other factor, nor was there any interaction. A power analysis showed that for the observed eccentricity effect to reach significance would require 314 subjects given the observed variance $\left(S^{2}=0.713\right)$, and that the observed flow effect would require 346 subjects given the observed variance $\left(S^{2}=0.202\right)$, implying that the risk of a Type II error in either case was low. For the eccentricity $\times$ flow interaction, the present experiment had sufficient power to detect a mean cell difference in $R$ of .08 , given the observed variance $\left(S^{2}=45.77\right)$. This suggests that coupling strength was strong and comparable in all flow conditions at all eccentricities.

Directional specificity. For every subject, there was a significant circular correlation between driver and sway directions, with a mean of $r=.67(p<.0001, N=720)$. In each condition, trials were significantly clustered about a mean direction rather than being randomly distributed (Table 2, column 3). Mean sway direction was $12.90^{\circ}$ $(S D=41.45)$ for $0^{\circ}$ radial flow, $66.26^{\circ}(S D=50.27)$ for $45^{\circ}$ intermediate flow, and $84.18^{\circ}(S D=43.78)$ for $90^{\circ}$ lamellar flow [Watson-Williams $F(2,717)=446.23, p<$ $.001]$. In addition, the $95 \%$ confidence interval of observed sway contained the specified driver direction in all lamellar conditions and in all but one radial condition (at $45^{\circ}$ eccentricity). However, in three of the four intermediate $45^{\circ}$ flow conditions, the direction of sway was biased toward the $90^{\circ}$ driver direction (i.e., parallel to the screen), in the direction of lamellar flow. Thus, responses were not directionally specific with $45^{\circ}$ flow displays. Note that this was due to the structure of the flow pattern, not to the retinal eccentricity, for a similar bias occurred at eccentricities of $0^{\circ}, 45^{\circ}$, and $60^{\circ}$ (there was only a nonsignificant trend in this direction at the $90^{\circ}$ eccentricity). We believe that this bias was due to triangulation errors with restricted samples of the flow field (see the General Discussion).

Driver sway phase. In each condition, trials were significantly clustered around a mean phase, with Raleigh tests ranging from $r=.58$ to $r=.93(N=72, p \mathrm{~s}<.05$ or better) (Table 2, columns 9 and 11). Mean phase was close to $-20^{\circ}$ and significantly different from zero in every condition. Mean phases were similar in the radial $(M=$ $\left.-19.14^{\circ}, S D=44.21\right)$, intermediate $\left(M=-19.56^{\circ}, S D=\right.$ 31.69), and lamellar $(M=-17.97, S D=34.90)$ flow conditions [Watson-Williams $F(2,717)=0.01, p>.05$ ]. This provides further evidence that sway was driven in a similar manner by different flow patterns. Further, unlike in Experiment 1, no statistical differences in mean phase were found between eccentricity conditions [Watson-Williams $F(3,716)=0.32, p>.05]$; the central mean phase of $\phi=$ $-17.1^{\circ}$ was half that of the comparable central condi- 
tions in Experiment $1\left(\phi=-33.2^{\circ}\right)$. Again, this was likely due to the addition of a fixation point in the central condition, so that stabilized gaze may have allowed the visual system to be more sensitive to display phase.

In sum, these results replicate and extend those of Experiment 1 by showing that a $22^{\circ}$ patch of optic flow can significantly influence postural sway at retinal eccentricities ranging from $0^{\circ}$ to $90^{\circ}$. Responses were directionally specific at all eccentricities with radial and lamellar flow, and even the directional bias observed in the intermediate flow condition was consistent across eccentricity. Furthermore, we found no evidence of differences in sway amplitude, cross-correlation, or phase as a function of eccentricity, and the power analyses indicated that the probability of erroneously accepting these null hypotheses was low. This pattern of similar adaptive responses across a wide range of eccentricities is consistent with the retinal invariance hypothesis.

\section{GENERAL DISCUSSION}

The present data indicate that the structure of optic flow is more important than the retinal locus of stimulation for controlling posture during locomotion. The main result of both experiments is that postural responses are functionally specific to radial, intermediate, and lamellar flow patterns, whether they appear in central or peripheral vision. Compensatory sway was strongly coupled to the driver, directionally specific, isotropic over driver direction, with large amplitudes and a constant phase, at all retinal eccentricities. The conclusion of functionally specific responses is warranted, first of all, by the fact that sway amplitudes and cross-correlations were statistically greater than control levels at all eccentricities. Second, it is supported by the finding that sway was directionally specific and covaried with driver amplitude at all eccentricities. The more difficult claim is that the sway amplitudes and cross-correlations were actually similar for all flow patterns at all eccentricities. We have obtained no evidence that postural responses to optic flow differ as a function of eccentricity, and the power analyses indicated that the likelihood of erroneously accepting the null hypothesis was small. These observations have obvious implications for the three self-motion hypotheses.

First, the results clearly contradict the hypothesis that peripheral vision dominates the perception and control of self-motion. Central stimulation $\left(0^{\circ}\right.$ eccentricity) elicited postural responses that were just as large and adaptive as those for peripheral stimulation $\left(30^{\circ}, 45^{\circ}, 60^{\circ}, 90^{\circ}\right.$ eccentricity). This finding is consistent with previous results demonstrating significant responses for standing posture in central vision (Andersen \& Dyre, 1989; Delorme \& Martin, 1986; Paulus et al., 1984; Stoffregen, 1985,1986 ), as well as centrally induced vection (Andersen \& Braunstein, 1985; Howard \& Heckmann, 1989; Post, 1988) and accurate central heading judgments
(Crowell \& Banks, 1993; Warren \& Kurtz, 1992). The peripheral dominance hypothesis must be rejected.

Second, the results are inconsistent with the functional sensitivity hypothesis, which states that the peripheral retina is insensitive (or markedly less sensitive) to radial flow for self-motion. We find that peripheral as well as central vision yields functionally specific postural responses to radial flow patterns. This is consistent with previous results showing accurate peripheral heading discrimination (Crowell \& Banks, 1993) and avoidance of looming objects (Stoffregen \& Riccio, 1990). However, it appears to be at variance with Stoffregen's $(1985,1986)$ observation that peripheral radial flow did not elicit postural sway during standing. What might account for the difference in our results?

A likely possibility is that our displays had larger amplitudes, and hence higher optical velocities, than did those of Stoffregen. Our driver amplitudes of 6.4 and $10.7 \mathrm{~cm}$ peak to peak yielded mean expansion rates of $0.026 / \mathrm{sec}$ and $0.044 / \mathrm{sec}$ over a half-cycle of oscillation. These are an order of magnitude larger than Stoffregen's, about $0.002 / \mathrm{sec}(1985$, Experiment 3 ) and $0.005 / \mathrm{sec}$ (1986, Experiment 2) ${ }^{4}$ Given that thresholds for motion detection and speed and direction discrimination all increase with eccentricity (Crowell \& Banks, 1996; McKee \& Nakayama, 1984; van de Grind, Koenderink, \& van Doorn, 1986), it is likely that Stoffregen's radial flow patterns were harder to detect in the periphery than ours, reflecting a general property of the visual system rather than a functional specialization. Consonant with this interpretation, reports of accurate peripheral heading discrimination (Crowell \& Banks, 1993) and avoidance responses (Stoffregen \& Riccio, 1990) also were based on much higher mean expansion rates, around $2.0 / \mathrm{sec}$ and $0.6 / \mathrm{sec}$, respectively. Although our expansion rates may be higher than those typical of standing sway, they are relevant to postural control during walking, when sway is greater.

Third, the present data support a retinal invariance hypothesis for postural control. The postural adjustments that we have observed are adaptive directional responses to the flow pattern regardless of its retinal eccentricity and even have similar amplitudes, cross-correlations, and phases in central and peripheral vision. This retinal invariance is similar to the recent data on heading judgments, with the exception of a narrow foveal advantage for radial flow patterns (Crowell \& Banks, 1993, 1996; Warren \& Kurtz, 1992). Why didn't we find this foveal advantage in postural responses? One possibility is that a foveal advantage that can be revealed by sensitive psychophysical tests may simply not show up in noisier postural responses. Another possibility is that, rather than a specialization for radial flow, the advantage is due to high acuity for the position and motion of elements near the FOE when it appears at the fovea. It is likely that such acuity would be degraded by large head movements dur- 


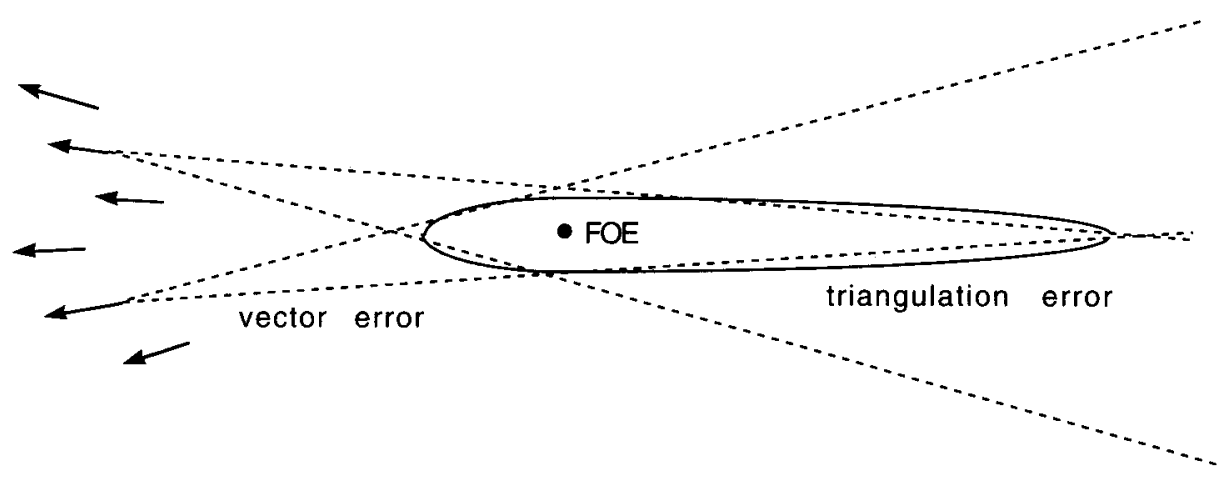

Figure 5. Schematic of triangulation error in locating the FOE from a restricted sample of intermediate flow, assuming noise in local motion extraction. Estimated FOE exhibits a constant bias.

ing locomotion. In either case, the import of our results is that the functional use of optic flow in the control of behavior appears to be retinally invariant.

Finally, it may seem puzzling that postural responses were directionally specific with radial and lamellar flow, but biased with the $45^{\circ}$ intermediate flow. We believe that this effect is attributable to the structure of the flow pattern, as follows. The required direction of sway is specified by the location of the FOE in the field of viewthe common point of intersection of all visible flow vectors. The visual system must, in effect, triangulate the visible flow vectors to locate the FOE. As Koenderink and van Doorn (1987) have pointed out, given that there is constant unbiased noise in extracting the direction of local velocity vectors, this can introduce triangulation errors in localizing the FOE (see Figure 5). Specifically, as the flow pattern is sampled farther from the FOE, the error in localizing the common point of intersection will increase, and a constant bias toward the opposite side of the true FOE will also increase. Thus, a pure radial pattern will yield minimum variable error and no bias, but as intermediate flow patterns become more lamellar, both the variable error and constant bias will increase. Indeed, when Crowell and Banks (1993, 1996) asked subjects to discriminate the headings of two successive flow patterns (thus measuring only the variable error), they found low errors with pure radial patterns (threshold $=$ $0.2^{\circ}$ with a $0^{\circ}$ radial flow) that increased as the flow became more lamellar (threshold $=3^{\circ}$ with a $30^{\circ}$ intermediate flow). In our postural data, a constant bias does not show up with a $30^{\circ}$ intermediate flow, but appears with a more lamellar $45^{\circ}$ intermediate flow pattern in the expected direction. The fact that a reliable bias recurred at three eccentricities $\left(0^{\circ}, 45^{\circ}, 60^{\circ}\right)$, with a consistent trend at the $90^{\circ}$ eccentricity, only reinforces the visual system's dependence on the structure of the flow pattern over the retinal region of stimulation.

In sum, our results favor the retinal invariance hypothesis that both central and peripheral vision can use radial and lamellar flow to control posture during walking. This is what one might expect from an adaptive postural system, for it is desirable that balance be adequately stabilized regardless of where the observer is looking. Control principles for posture during locomotion thus appear to be based primarily on the structure of the optic flow pattern, regardless of its retinal eccentricity.

\section{REFERENCES}

Amblard, B., \& Carblanc, A. (1980). Role of foveal and peripheral vision information in the maintenance of postural equilibrium in man. Perceptual \& Motor Skills, 51, 903-916.

ANDERSEn, G. J., \& Braunstein, M. L. (1985). Induced self-motion in central visıon. Journal of Experimental Psychology: Human Perception \& Performance, 11, 122-132.

ANDERSEN, G. J., \& Dyre, B. P. (1989). Spatial onentation from optic flow in the central visual field. Perception \& Psychophysics, 45, 453-458.

Bardy, B. G., Warren, W. H., \& KaY, B. A. (1996). Motion parallax is used to control postural sway during walking. Experimental Brain Research, 111, 271-282.

Batschelet, E. (1981). Circular statistics in biology. New York: Academic Press.

Berthoz, A., Pavard, B., \& Young, L. R. (1975). Perception of linear horizontal self motion induced by peripheral vision (linear vection). Experimental Brain Research, 23, 471-489.

Brandt, T., Dichgans, J., \& Koenig, E. (1973). Differential effects of central versus peripheral vision on egocentric and exocentric motion perception. Experimental Brain Research, 16, 476-491.

Buser, P., \& Imbert, M. (1987). Vision. Paris: Hermann.

Crowell, J. A., \& Banks, M. S. (1993). Perceiving heading with different retınal regions and types of optic flow. Perception \& Psychophysics, 53, 325-337.

Crowell, J. A., \& Banks, M. S. (1996). Ideal observer for heading judgments. Vision Research, 36, 471-490.

Delorme, A., \& MarTin, C. (1986). Roles of retınal periphery and depth periphery in linear vection and visual control of standing in humans. Canadian Journal of Psychology, 40, 176-187.

Dichgans, J., \& BrandT, T. (1978). Visual-vestibular interaction: Effects on self-motion perception and postural control. In R. Held, H. Leibowitz, \& H.-L. Teuber (Eds.), Handbook of sensory physiology Vol 8. Perception (pp. 755-804). New York: Springer-Verlag.

Dukstra, T M. H., Schöner, G., \& Gielen, C. C. A. M. (1994). Temporal stability of the action-perception cycle for postural control in a moving visual environment. Experimental Brain Research, 97, 477-486. 
Ferrigno, G., \& Pedotti, A. (1985). EliTE: A digital dedicated hardware system for movement analysis via real time TV-signal processing. IEEE Transactions on Biomedical Engineering, 32, 943-950.

GiBSON, J. J. (1950). The perception of the visual world. Boston: Houghton Mifflin.

GiBSon, J. J. (1968). What gives rise to the perception of motion? Psychological Review, 75, 335-346.

Granat, M. H., BarnetT, R. W., Kirkwood, C. A., \& Andrews, B. J. (1991). Technique for calculating the direction of postural sway. Medical \& Biological Engineering \& Computing, 29, 599-601.

Held, R., Dichgans, J., \& BAUER, J. (1975). Characteristics of moving visual areas influencing spatial orientation. Vision Research, 15, 357-365.

Howard, I. P., \& HeckmanN, T. (1989). Circular vection as a function of the relative sizes, distances, and positions of two competing visual displays. Perception, 18, 657-665.

KePPEL, G. (1982). Design and analysis: A researcher's handbook. Englewood Cliffs, NJ: Prentice-Hall.

KoEnderink, J. J., \& VAN DoORn, A. J. (1987). Facts on optic flow. Biological Cybernetics, 56, 247-254.

Lestienne, F., Soechting, J., \& Berthoz, A. (1977). Postural readjustments induced by linear motion of visual scenes. Experimental Brain Research, 28, 363-384.

MARPLE, S. L. (1986). Digital spectral analysis with applications. Englewood Cliffs, NJ: Prentice-Hall.

MCKeE, S. P., \& Nakayama, K. (1984). The detection of motion in the peripheral visual field. Vision Research, 24, 25-32.

Nougier, V., Bard, C., Fleury, M., \& Teasdale, N. (1997). Contribution of central and peripheral vision to the regulation of stance. Gait \& Posture, 5, 34-41.

Paulus, W., Straube, A., \& Brandt, T. (1984). Visual stabilization of posture: Physiological stimulus characteristics and clinical aspects. Brain, 107, 1143-1163.

Paulus, W., Straube, A., Krafczyk, S., \& Brandt, T. (1989). Differential effects of retinal target displacement, changing size, and changing disparity in the control of anterior/posterior and lateral body sway, Experimental Brain Research, 78, 243-252.

Pirenne, M. H. (1967). Vision and the eye (2nd ed.). New York: Methuen.

PosT, R. B. (1988). Circular vection is independent of stimulus eccentricity. Perception, 17, 737-744.

STOFFREGEN, T. A. (1985). Flow structure versus retinal location in the optical control of stance. Journal of Experimental Psychology: Human Perception \& Performance, 11, 554-565.

STOFFREGEN, T. A. (1986). The role of optical velocity in the control of stance. Perception \& Psychophysics, 39, 355-360.
Stoffregen, T. A., \& Riccio, G. E. (1990). Responses to optical looming in the retinal center and periphery. Ecological Psychology, 2, 251-274.

van der Grind, W. A., Koenderink, J. J., \& VAN DoOrn, A. J. (1986). The distribution of human motion detector properties in the monocular visual field. Vision Research, 26, 797-810.

WARREN, W. H., KaY, B. A., \& YilmaZ, E. H. (1996). Visual control of posture during walking: Functional specificity. Journal of Experimental Psychology: Human Perception \& Performance, 22, 818-838.

WARREN, W. H., \& KuRTZ, K. J. (1992). The role of central and peripheral vision in perceiving the direction of self-motion. Perception \& Psychophysics, 51, 443-454.

\section{NOTES}

1. Even though a sinusoidal driver may be anticipated by the subject, to do so in a functionally specific manner requires that the flow pattern be accurately detected in central or peripheral vision and used to control sway adaptively. This is precisely what we wished to determine. Further, a current view holds that matching the temporal characteristics of the stimulation, which is often self-produced, may be more basic to postural control than responding to a random driver (Dijkstra, Schöner, \& Gielen, 1994).

2. In Experiment 1, a fixation point was not used in the central condition, to avoid relative motion between it and the wall display, including induced motion of the fixation marker that might affect sway. Experiment 2 replicated the findings of Experiment 1, but with a fixation point in the central condition.

3 . The multiple $R$ provides an overall measure of the similarity between the driver and sway, since it is sensitive to phase, amplitude, and waveform fluctuations during a trial. An alternative measure, the magnitude squared coherence (MSC; e.g., Marple, 1986), may provide a more refined measure of the linear coupling of two signals, because it is sensitive to only phase and amplitude fluctuations; however, the MSC is statistically unreliable with the short data records of this study.

4. The expansion rate for Stoffregen (1985) is calculated from the experimental parameters, $\theta=60^{\circ}, F=0.08 \mathrm{~Hz}$, and $A=2.5 \mathrm{~cm}$ with a surface at a distance of about $1.85 \mathrm{~m}$. The total change in visual angle in one half-period $(6 \mathrm{sec})$ is $\Delta \theta=0.67^{\circ}$, and the mean expansion rate is $\Delta \theta / 6 \theta=0.002 / \mathrm{sec}$. The expansion rate for Stoffregen (1986) is determined from his Figure 4.

(Manuscript received July 17, 1996; revision accepted for publication July 17,1998 .) 